\title{
Civilisations
}

Revue internationale d'anthropologie et de sciences

humaines

$41 \mid 1993$

Mélanges Pierre Salmon II

\section{Incident de frontière au Kivu}

\section{André Lederer}

\section{(2) OpenEdition \\ Journals}

Édition électronique

URL : http://journals.openedition.org/civilisations/1730

DOI : $10.4000 /$ civilisations. 1730

ISSN : 2032-0442

\section{Éditeur}

Institut de sociologie de l'Université Libre de Bruxelles

\section{Édition imprimée}

Date de publication : 1 septembre 1993

Pagination : 415-426

ISBN : 2-87263-094-5

ISSN : 0009-8140

\section{Référence électronique}

André Lederer, « Incident de frontière au Kivu », Civilisations [En ligne], 41 | 1993, mis en ligne le 30 juillet 2009, consulté le 10 décembre 2020. URL : http://journals.openedition.org/civilisations/1730 ; DOI : https://doi.org/10.4000/civilisations. 1730

Ce document a été généré automatiquement le 10 décembre 2020.

(C) Tous droits réservés 


\title{
Incident de frontière au Kivu
}

\author{
André Lederer
}

\section{La frontière de l'Etat Indépendant du Congo au Kivu}

1 En marge de la Conférence de Berlin, l'Acte de Neutralité avait défini les frontières de l'Etat Indépendant du Congo. Or, en 1885, la portion nord-est du nouvel Etat était mal connue. Si le lac Tanganika avait été découvert en 1857 par Burton et Speke et le lac Edouard en 1876 par Stanley, le lac Kivu ne le fut qu'en 1894 par le comte von Götzen, soit neuf ans après la signature de l'Acte de Neutralité.

2 En l'absence de toute précision géographique dans ces régions, la limite du nouvel Etat avait été définie comme suit: "une droite issue de l'extrémité nord du lac Tanganika et aboutissant en un point situé sur le $30^{\mathrm{e}}$ méridien est et à $1^{\circ} 20^{\prime}$ de latitude sud; plus au nord, la frontière est constituée par le $30^{\mathrm{e}}$ méridien est". Il en résultait que les deux rives de la Ruzizi et du lac Kivu, dont on ignorait l'existence, étaient entièrement en territoire de l'E.I.C. ${ }^{1}$.

En 1884, les Allemands s'intéressèrent aux Colonies dont, notamment, l'Afrique Orientale; ils firent de Tabora leur capitale et créèrent des villes comme Dar-es-Salaam, Kigoma, Usumbura et d'autres.

Tout se passa bien jusqu'en 1896, lorsque la révolte de la colonne Dhanis, en route pour le Nil, obligea les Belges à évacuer la portion de territoire comprise entre la frontière officielle et la vallée de la Ruzizi et du lac Kivu. En octobre 1899, les Belges, ayant défait les mutins, ne purent réoccuper certains postes, les Allemands prétendant s'y maintenir. Léopold II, désirant conserver les deux rives de la Ruzizi et du Kivu, fit créer, en 1904, une commission mixte qui devait oeuvrer sur le terrain².

Pour renforcer la position belge, Costermans, commandant militaire de la région, fit fonder par le commandant Olsen un poste à Nya-Lukemba, l'actuel Bukavu, le $1^{\text {er }}$ juillet 1900. D'un autre côté, les Allemands avaient fait un accord avec les Anglais, de façon à obtenir tout le massif du Kilimandjaro, à la frontière entre l'Uganda et l'Est-Africain 
allemand, donnant en échange aux Britanniques l'Ufumbiro. Mais où se situait-il ? Dans son livre "The journal of the discovery of the sources of Nile", Speke donne un dessin montrant dans le lointain les Monts Mfumbiro, mais l'endroit où les Allemands envoyaient les Anglais était la plaine de l'Ufumbiro. Confusion volontaire ou non à cause de la quasi-homonymie ? ${ }^{3}$.

\section{L'incident de l'Ufumbiro}

Le 26 juin 1909, J.M. Coote, commissaire de district de M'Barara, en Uganda, adressa une lettre du Mont Lubuna à F. Goffoel, chef de secteur de Rutshuru, annonçant que, conformément aux instructions reçues, il venait occuper l'Ufumbiro. Dorénavant, la limite de son district se situait à $29^{\circ} 47^{\prime}$ est au nord de $1^{\circ} 20^{\prime}$ de latitude sud, et non plus au $30^{\mathrm{e}}$ méridien. Il en résultait que Rutshuru appartenait au district de M'Barara et il invitait le commandant Olsen à le rencontrer à Lubuna en vue de conclure un accord, en attendant une décision à prendre par les gouvernements ${ }^{4}$.

7 Coote, pénétrant avec une troupe au Congo en direction sud-ouest, suivit une bande de territoire indiquée sur une carte par les Allemands et attribuée par ceux-ci aux Anglais, de façon à leur donner un accès direct au lac Kivu, sans traverser le territoire belge ${ }^{5}$.

8 L'avance avait été rapide, plusieurs facteurs ayant joué en faveur de Coote : région peu occupée car non encore ouverte au commerce et à l'industrie, pas de reconnaissance belge au sud de Rutshuru, mauvaise volonté de certains chefs indigènes qui n'avaient pas signalé à l'autorité belge le passage de troupes étrangères.

9 En outre, il faut reconnaître que Coote avait mené son expédition avec astuce : rapidité de la préparation, secret de l'opération, avance par bonds rapides et uniquement de nuit.

10 Après avoir reconnu la rive du lac Kivu près de Coma, les Anglais installaient deux camps fortifiés, l'un à Burungu, l'autre au Mont Lubuna ${ }^{5}$.

11 Olsen avait toujours veillé à la formation de ses subordonnés et à faire régner une forte discipline dans les troupes sous ses ordres. Aussi, la réaction de Goffoel fut excellente. Au reçu de la lettre de Coote, il lui fait savoir qu'il n'a pas qualité pour traiter pareille question et qu'il a transmis sa demande au commandant Olsen, commandant supérieur des territoires de la Ruzizi-Kivu, à ce moment en tournée d'inspection dans la région de Kasindi, au nord du lac Edouard ${ }^{6}$; il informe également son supérieur, O. Baudelet, chef de la zone de Beni-Rutshuru?

D'après les indigènes porteurs du message de Coote, deux Européens et une troupe de 50 hommes se trouvent à 3 heures de marche au sud-est de Rutshuru, donc en territoire nettement congolais. Pour vérifier ces dires, il envoie une mission dirigée par le capitaine Wangermée et le lieutenant Brochard, accompagnés de 100 hommes. Copie de toute cette correspondance est adressée au commandant Olsen ${ }^{8}$.

13 Wangermée rencontra Coote près de Bayanza; il eut envie d'enlever le drapeau anglais arboré au mât du camp, mais il n'osa accomplir ce geste par crainte des répercussions qu'il aurait entraîné.

14 Wangermée remit le message de Goffoel à Coote, lui disant qu'il n'avait aucune qualité pour le discuter, mais il engagea l'officier anglais à ne plus avancer au-delà du point où il était arrivé9. Coote acquiesça, puis ils se mirent à converser amicalement; l'Anglais conclut que le point où il se trouvait était reconnu en zone britannique par les Belges. En 
conséquence, affirmait l'Anglais, le territoire objet de l'accord était devenu territoire contesté et aucune des deux puissances ne pouvait y faire acte d'autorité ${ }^{10}$.

Wangermée de son côté fit savoir qu'il avait protesté et, qu'à son retour, il avait appris du chef Bayanza que Coote avait interdit qu'il approvisionne Rutshuru en vivres. Wangermée fit observer au chef Bayanza que son territoire était toujours sous dépendance belge et qu'il avait à approvisionner Rutshuru, comme auparavant ${ }^{11}$.

\section{Réaction d'Olsen}

De son côté, Olsen, qui se trouvait à Kasindi, ne reçut la lettre de Coote que le $1^{\mathrm{er}}$ juillet 1909. Immédiatement, il envoya au gouvernement belge un télégramme déposé à Fort Portai, en Uganda, dont voici la teneur: "Violation territoire belge par Anglais établie Ufumbiro. Commissaire anglais annonce qu'il procède occupation et administration Ufumbiro. Anglais prétendent Rutshuru situé territoire anglais. Je me dirige sur le camp anglais Ufumbiro ; troupes pour me précéder. Emploierai tous moyens, même force armée pour obliger Anglais respecter notre frontière. Commandant supérieur Ruzizi-Kivu ${ }^{12}$.

Le 2 juillet 1909, Olsen envoyait à Coote une lettre (voir annexe) dans laquelle il disait être très étonné du contenu de la lettre de Coote qui est en contradiction avec l'accord mis au point en avril 1904 par une commission mixte anglo-congolaise. Ainsi, il protestait de la façon la plus énergique contre la violation d'un territoire incontestablement belge par une troupe anglaise (il faut savoir que c'est seulement le 27 juin 1913 que l'Angleterre reconnut l'E.I.C. comme Congo belge). Olsen se sent d'autant plus fort que le Ministre Britannique à Bruxelles a fait savoir au Gouvernement belge que les fonctionnaires anglais de l'Uganda ont été prévenus qu'ils avaient à s'en tenir strictement à l'accord de 1904. Si vraiment, le Gouvernement anglais avait eu l'intention d'occuper l'Ufumbiro, il eut été plus loyal d'en aviser le Gouvernement belge ${ }^{13}$.

\section{Le face à face de dix mois}

Coote ne voulait pas céder et, de son côté, Olsen n'acceptait aucune rencontre en territoire belge ; la question devait être examinée par les gouvernements respectifs.

Olsen était le plus fort dans cette situation d'attente, car il disposait sur le territoire belge de villages qui approvisionnaient les troupes de l'E.I.C., tandis que Coote avait été stoppé dans la zone marécageuse entre les lacs Mutanda, Bunioni et Muleira. Il disposait de 8 officiers, 350 hommes, 2 canons. Il n'attaquera pas mais défendra à outrance la position qu'il occupe.

En juillet et août 1909, une série d'incidents vont se succéder entre les Congolais et les Anglais, puis les Allemands et ce fait ne semble pas étranger à la présence simultanée au Congo du Prince Albert et du Ministre Renkin en tournée dans le Congo, devenu belge depuis le 18 octobre 1908, ce que l'Angleterre ne reconnaît qu'en $1913^{14}$. 


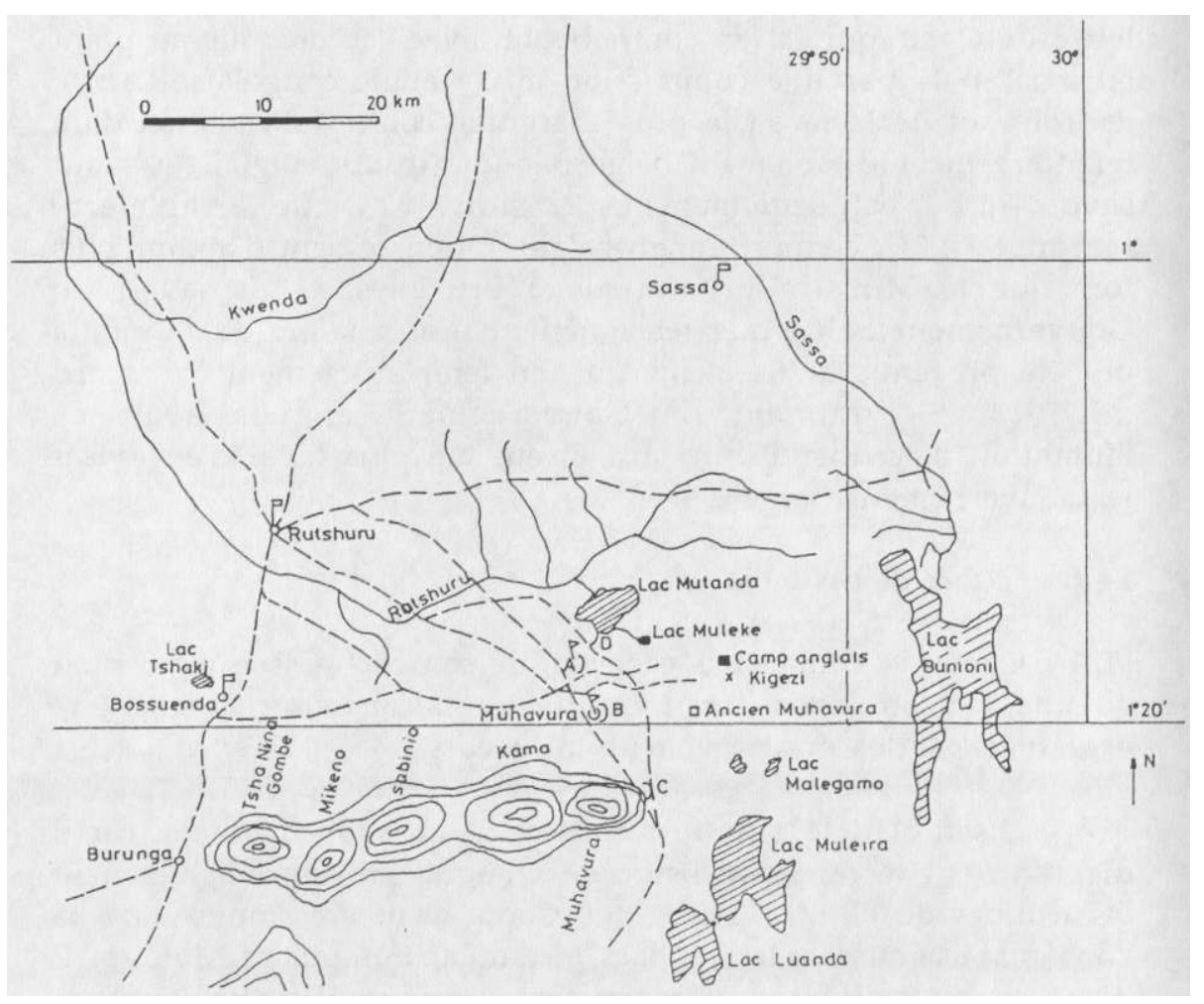

Des discussions s'élèvent, notamment au sujet de blessures survenues à des indigènes pour savoir si elles proviennent de flèches ou de balles de fusil. Alors qu'Olsen faisait faire les constats par le docteur Coronisio, un médecin italien de valeur, Coote envoyait le lieutenant Mac Combie, qui avait une grande expérience des blessures, car il avait pris part à la guerre des Boers ; en réalité, ce n'était qu'un commissaire de police ${ }^{15}$.

Les Anglais ne manquaient aucune occasion de créer des incidents dont ils essayaient de faire endosser la responsabilité aux soldats du Congo belge. Olsen n'attaque pas, mais ne laisse pas passer les Anglais. De leur côté, les Allemands installaient des colons et créaient des postes à la rive est de la Ruzizi et du lac Kivu, sans que les Belges ne s'en inquiètent outre mesure, Léopold II ayant reconnu la zone d'influence anglaise décrite dans l'arrangement anglo-allemand du 27 juillet 1890, d'autant plus que l'Angleterre cédait à bail l'enclave de Lado à l'Etat Indépendant, ce qui comblait les rêves nilotiques de Léopold II. En échange, l'Etat Indépendant cédait à l'Angleterre une bande de $25 \mathrm{~km}$ de large du lac Tanganika au lac Edouard, ce qui leur permettait la réalisation du grand projet d'un chemin de fer du Cap au Caire.

L'Allemagne voyait d'un mauvais oeil la politique d'expansion de l'Angleterre et s'unit à la France qui s'opposait, de son côté, à l'accès de l'Etat Indépendant au Haut-Nil. La politique de l'Allemagne se modifia à partir de 1894 ; jusqu'alors, elle avait laissé les mains libres à Léopold II dans la région du Haut-Nil, dont on ignorait la géographie. Bismarck, qui avait joué le jeu de Léopold II et de l'A.I.C, se berçait du secret espoir que cet Etat ne serait pas viable ${ }^{16}$.

Pendant le long face à face avec les Anglais, les Allemands créaient aussi des difficultés dans le territoire contesté au nord du lac Kivu. Dans une région frontière où les Allemands avaient l'exercice de la souveraineté, un missionnaire français, le R.P. Loupias, avait été assassiné le $1^{\mathrm{er}}$ avril 1910 au poste de Ruaza ${ }^{17}$. Olsen, dont les troupes étaient les plus proches de ce point, envoie sans hésiter ses hommes rétablir l'ordre. Les Allemands, 
quoique vexés, furent obligés de le remercier ; malgré la gravité de la situation, Olsen ne manquait pas d'humour ${ }^{18}$.

\section{La diplomatie entre en action}

Le problème des frontières dans la région du Kivu devait être réglé diplomatiquement pour éviter les incidents sur le terrain.

Aussi, le 8 février 1910, à Bruxelles, se réunirent les délégués des puissances; pour l'Allemagne, Monsieur Ebermayer et le baron von Danckelman, pour l'Angleterre, Sir A. Hardinge, M. Tilley et le colonel Close et, pour la Belgique, le ministre d'Etat Van den Heuvel, M. Van Maldeghem et le chevalier van der Elst. MM. de Bassompierre et Orts, assuraient le secrétariat. Les séances se tenaient à huis-clos et le secret était bien gardé ${ }^{19}$.

27 Le but de ces réunions était de substituer les lignes déterminées arbitrairement pour former les frontières par des limites correspondant à des réalités géographiques. Alors que Léopold II était intraitable à ce sujet, le roi Albert montrait plus de souplesse pour accepter des concessions réciproques; par sa clairvoyance et sa droiture, il avait conduit les délégués à trouver une solution unanimement acceptée. Ainsi, le 14 mai 1910, le protocole d'accord était signé par les délégués des trois puissances ${ }^{20}$.

B Gâce à la fermeté d'Olsen, le travail des diplomates belges fut facilité. Olsen, de son côté, fit aussi montre d'un sérieux talent diplomatique dans la correspondance qu'il échangea avec les Anglais et les Allemands. En récompense des services rendus, il fut nommé chevalier de l'Etoile Africaine ${ }^{21}$.

29 Le traité signé le 14 mai 1910 mettait fin définitivement à tous les conflits de frontière dans cette région. Seule restait à déterminer sur le terrain la position $\mathrm{du} 30^{\mathrm{e}}$ méridien, ce qui dura plusieurs années.

\section{ANNEXES}

$\mathrm{N}^{\circ}$ 396. Kasindi, le 2 juillet 1909

Monsieur le Commissaire de district,

J'ai l'honneur de vous accuser réception de votre honorée lettre datée Lubuna le 26 juin 1909 et c'est avec le plus grand étonnement que j'ai pris connaissance de son contenu.

Conformément à l'arrangement du 12 mai 1894 intervenu entre nos deux Gouvernements, la frontière entre le Protectorat de l'Uganda et le Congo Belge dans la région Ufumbiro qui fait l'objet de votre lettre, est constituée par le $30^{\mathrm{e}}$ méridien Est de Greenwich. Ce $30^{\mathrm{e}}$ méridien vrai a été déterminé par une commission mixte anglo-congolaise en 1907-08 et, en vertu de l'accord d'avril 1904 conclu entre l'Angleterre et l'Etat Indépendant du Congo, aujourd'hui Congo Belge, la zone située entre le $30^{\mathrm{e}}$ méridien primitivement supposé (méridien Mac Donald) est provisoirement neutralisée. Il est interdit aux fonctionnaires des deux Gouvernements de faire acte d'occupation dans ce territoire neutre. 
Immédiatement à l'Ouest du méridien Mac Donald, limite occidentale de la bande neutre, le territoire est incontestablement belge. Ce territoire est occupé et administré par nous depuis plus de 10 ans sans avoir soulevé des objections des autorités anglaises.

Je proteste donc de la façon la plus énergique contre votre violation d'un territoire nettement belge par une force armée anglaise.

Depuis plus de 10 ans que je séjourne dans la région frontière voisine du $30^{\mathrm{e}}$ méridien, c'est la première fois que je vois émettre des prétentions anglaises telles que vous les exposez dans votre lettre et je suis à me demander s'il n'y a pas erreur de votre part dans l'application des revendications du Gouvernement anglais. Je suis d'autant plus étonné des actes que vous posez que tout récemment, comme suite à une communication de son Excellence le Ministre de Sa Majesté Britannique à Bruxelles, mon Gouvernement m'a informé de ce que les fonctionnaires anglais de l'Uganda avaient été rappelés à une stricte observance des prescriptions de l'accord de 1904.

Non seulement vous vous mettez en flagrante contradiction avec les accords précités en vous installant en bande neutre, mais vous pénétrez en territoire incontestablement belge que vous prétendez administrer au nom du Gouvernement britannique.

J'ai peine à croire que le Gouvernement anglais émette de pareilles prétentions qu'aucun acte antérieur ne permet de défendre. Mais cependant si, en violation des traités et accords conclus, le Gouvernement Britannique manifestait l'intention d'occuper l'Ufumbiro, territoire nettement belge et administré par nous depuis plus de 10 ans, il eut été plus loyal, plus conforme aux usages, de s'adresser directement au Gouvernement Belge à Bruxelles.

Je n'ai personnellement aucune qualité pour conclure de nouveaux arrangements avec le Gouvernement Britannique. Ma mission se borne à la défense des droits acquis et des intérêts de mon Gouvernement et notamment de maintenir le respect de notre frontière. Cette mission dont $\mathrm{j}$ 'assume l'entière responsabilité envers mon Gouvernement constitue pour moi le devoir le plus impérieux et le remplirai jusqu'au bout.

Je considère votre mouvement en territoire belge comme une action hostile et je ne puis donc avoir aucune entrevue avec vous aussi longtemps que vous vous trouverez à l'Ouest de la bande neutre. Si malgré les avis que je vous donne, vous maintenez votre occupation en territoire nettement belge, le Gouvernement Britannique devra supporter la grande responsabilité des moyens que bien à regret je devrai employer pour vous ramener au respect du territoire ressortissant à la Colonie Belge. J'ai toutefois la plus grande confiance en votre sagesse, Monsieur le Commissaire de district, et j'ai le ferme espoir que dès le reçu de la présente lettre vous reporterez votre occupation à l'Est de la bande neutre laissant ainsi à nos gouvernements respectifs le soin de régler les questions litigieuses. Je crois inutile d'attirer encore votre attention sur l'interdiction d'occuper la bande neutre ; c'est un principe établi par l'accord de 1904 qui vient d'être rappelé aux fonctionnaires des deux parties qui a toujours été ponctuellement observé par nous.

Je vous exprime encore mes regrets de ne pouvoir vous accorder l'entrevue que vous me demandez; je subordonne ma ligne de conduite à la vôtre ; mais j'ose espérer, Monsieur le Commissaire de district, que vous ne vous ferez pas l'ouvrier d'incidents regrettables et de nature à troubler la paix des populations indigènes. 
J'ai l'honneur de vous prier, Monsieur le Commissaire de district, de vouloir bien agréer l'assurance de ma haute considération.

Le Commandant Supérieur,

(s.) Olsen.

\section{NOTES}

1. FLAMENT, F. : 1952, La Force Publique de sa naissance à 1914, Bruxelles, Mém. de l'IRCB, cl. sc. Mor. et Polit., sér. in 8 $8^{\circ}, 27,1,185-189$.

2. LEDERER, A. : Le rôle des flottes fluviale et lacustre au Congo Belge durant la première guerre mondiale, Bruxelles, 1988, Collectanea Maritima IV, byl. tot de intern. mar. gesch. Kon. Acad. W.L.S.K. 123.

3. SPEKE, J.H. : 1864, The Journal of the discovery of the sources of the Nile, Edimbourg et Londres, 214.

4. COOTE, J.M. à GOFFOEL, F. : Lettre de Mont Lubuna 26-6-1909, annexe lettre du G.G. au Min. CoL, p. 38, AE 345, arch. ex-M.A.A.

5. COOTE, J.M., sept. 1956, The Kivu Mission 1909-1910, The Uganda Journal, vol. 20, n 2, p. 106.

6. GOFFOEL, F. à COOTE, J.M. : Rutshuru, 27-6-1909, an. lettre Olsen au G.G., A.E. 345, p. 38, arch. ex-M.A.A.

7. GOFFOEL, F. à OLSEN, F.W. , op.cit., p. 38.

8. GOFFOEL, F. à OLSEN, F.W. , idem.

9. GOFFOEL, F. à WANGERMEE, G. : Rutshuru, 28-6-1909, an. 1 à lettre du G.G. au Min. col., Boma, 6-9-1909, A.E. 345, p. 51, arch. ex-M.A.A.

10. COOTE, J.M. à GOFFOEL, F. : Rubana-Nile, 29-6-1909, an. 4 à lettre G.G. au Min. Col. 6-9-1909, A.E. 345 , arch. ex-M.A.A.

11. WANGERMEE, G. à GOFFOEL, F. : Bayanza, 28-6-1909, an. 2 à lettre G.G. au Min. CoL, Boma, 6-9-1909, A.E., 345, p. 51, ex-M.A.A.

12. OLSEN, F.W. à Min. Col. : Kasindi, 2-7-1909, AE, 345.

13. OLSEN, F.W. à COOTE, J.M. : Kasindi, 2-7-1909, A.E. 345, p. 396 (lettre donnée en annexe).

14. BROCHART à GOFFOEL, F.: 19-9-1909 et carte annexe, A.E. 345 et RENKIN J. à M.A.E., Bruxelles, 10-8-1909, dossier A.E. 345, arch. M. A.E. ; COOTE, J.M. à OLSEN, F.W. Kigezi 19-8-1909, dossier A.E. 345, arch. M.A.E.

15. OLSEN, F.W. à COOTE, J.M. : Muhavura, 16.8.1909, dossier A.E., 345, arch. M.A.E. ; COOTE, J.M. à OLSEN, F.W. : Kigezi, 19.8.1909, dossier A.E. 345, Arch. M.A.E.

16. VAN VRACEM, p.: Recherches sur la formation de la frontière orientale du Congo belge, Bukavu et Bruxelles 1958, Inst. pour la recherche scientif. en Afrique centrale, onzième rap. an., pp. 135-137.

17. VANNESTE, P.M. ; Loupias, Paulin, Bruxelles, 1958, biogr. col. belge 2, col. 563-566.

18. LEDERER A. : Frederik Waldemar Olsen, Bruxelles, 1966, Bull. des s. de l'ARSOM, 1, 524, 525.

19. La frontière orientale, Bruxelles, 1910, Mouv. Géogr., col. 84.

20. La conférence de la frontière orientale du Congo belge, Bruxelles, 1910, Mouvement Géographique, col. 106-107 et 263-264.

21. LEDERER, A. : Frederik Waldemar Olsen, op.cit., p. 525. 


\section{RÉSUMÉS}

As part of the Berlin Conference, the Neutrality Act defined the frontiers of the independant state of the Congo. However, in 1885 the north-eastern part of the new state was still little known. In the absence of any geographical precision, the defined limits of the state included the two banks of the river Ruzizi and lake Kivu within the territory of the Congo. These innaccuracies led to diverse incidents at the frontier of Kivu from 1899, first with the Germans and then with the British, both of whom wished to settle on the banks of the lake. Again in July and August 1909, a series of incidents took place between the Congolais and the British and then the Germans. The scale of the problem and the desire to avoid an escalation necessitated a diplomatic solution to the Kivu border problem. Thus, in May 1910, an agreement was concluded between the Belgians, the Germans and the British. This agreement put a definite end to all the border conflicts in this region.

\section{AUTEUR}

\section{ANDRÉ LEDERER}

Université Catholique de Louvain - Belgique 